\title{
GALACTIC AND METAGALACTIC MAGNETIC FIELDS
}

\author{
K. BRECHER \\ University of California, San Diego, Calif., U.S.A.
}

\begin{abstract}
All of the possible sources of primeval magnetic fields are examined and found to be inadequate to account for the observed galactic $B$-fields (if the magnetic fields in galaxies are the direct result of the condensation of a magnetized metagalactic medium). Field amplification in dense rotating objects (perhaps neutron stars or galactic nuclei) may be responsible for the bulk of such fields. Most of the (hypothetical) intergalactic $B$-field then originates in galaxies and is dragged along with the cosmic rays escaping from them. Limits are placed on the value of any present intergalactic magnetic field of either primordial or 'recent' origin.
\end{abstract}

\section{Introduction}

In trying to understand the origins and dynamical importance of cosmic magnetic fields, one would like to answer the following two questions: First, how do the metagalactic and galactic magnetic fields, $B_{\text {met }}$ and $B_{\text {gal }}$, evolve in time? Second, what is the spatial connection between $B_{\text {met }}$ and $B_{\text {gal }}$ ? In order to answer the first of these, the theoretically and observationally allowable magnitudes of the fields must be unambiguously determined. We may then understand whether one gives rise directly to the other, or whether their temporal evolution is more complicated (e.g. if a small metagalactic seed field is trapped in the material forming a protogalaxy, amplified by galactic processes, and subsequently ejected to fill intergalactic space.) A knowledge of the topology of the fields is essential to determining what role, if any, they play in determining galactic structure or, conversely, what role the properties of discrete sources play in determining them.

\section{Primordial Magnetic Fields}

Because of the difficulty of accounting for the observed galactic magnetic fields by conventional means, it has become common to consider them as primordial (Woltjer, 1965), a remnant of some earlier stage either of the universe or of their own development in protogalaxies. Though little is known of the latter, the existence of primeval $B$-fields may be examined within the framework of evolving relativistic cosmological models. Let us, therefore, consider the sources of magnetic fields in the ylem and the assumed uniform metagalactic medium which could give rise to the magnetic fields in normal galaxies of order $B_{\mathrm{gal}} \approx 5 \times 10^{-6} \mathrm{G}$ by direct condensation (without amplification). If the intergalactic medium is ionized (it need not be fully ionized), the magnetic field is 'frozen' into the matter, and for simple condensation of the medium into a galaxy, one must have a present metagalactic field of $B_{0} \approx 10^{-9} q_{0}^{1 / 2} \mathrm{G}$ where $q_{0}$ is the deceleration parameter for the model. ( $B_{0}$ can also depend weakly on the redshift $z$ of galaxy formation for $z \lesssim 10$.) In steady state cosmology, magnetic fields (vectors) could presumably form spontaneously along with matter (scalar). 
The sources which could in principle support a primeval magnetic field are: (i) purely random thermal and small scale turbulently generated currents; (ii) displacement currents (i.e., time varying $E$-fields with respect to co-moving co-ordinates); (iii) ordered currents arising from vorticity or density fluctuations; (iv) aligned dipole moments of baryons and leptons; (v) magnetic monopoles; and (vi) source free fields (i.e. solutions to the Einstein-Maxwell equations which are homogeneous, $\mathbf{B}(\mathbf{r}, t)=\mathbf{B}(t)$, and without currents, $\nabla \times \mathbf{B}=0$ everywhere).

In a recent paper (Brecher and Blumenthal, 1970), upper limits have been set on the first four by thermodynamic and electrodynamic arguments. For example, in the absence of large density fluctuations $(\delta \varrho / \varrho \ll 1)$, a very high upper limit on any primordial $B$-field surviving to the present may be set of order $B_{0} \lesssim 5 \times 10^{-13} q_{0 /}^{12} \mathrm{G}$ (from the evolution of the thermal energy density of the fluid). In Batchelor's vorticity analogy for determining the final strength of turbulently generated magnetic fields, this result would be reduced by $R^{1 / 4}$, where $R \gg 1$ is the Reynolds number of the turbulent flow. Of course, these considerations apply only to homogeneous, isotropic, steady, incompressible turbulence. Before $z \sim 10^{3}$, one should in fact consider the properties of an expanding radiation-dominated compressible medium for which there is no theory at present.

Two papers dealing with fluctuations in the early universe, however, have recently arrived at different conclusions regarding primordial $B$-fields. Harrison (1970) has suggested that galaxies arise in dense, spinning cores in the early universe. The existence of large density fluctuations in this case serves as the necessary energetic source for the fields. By arbitrarily postulating complex initial conditions, it is not surprising that magnetic fields can also arise in the early universe.

Ozernoy and Chernin (1968) have considered the existence of what they call 'photon turbulence', as the necessary 'external' source of energy which can drive the metagalactic matter turbulence to generate magnetic fields. In the era $z>10^{4}$, they specify that the turbulent photon energy is much greater than either the matter or thermal radiation energy densities. However, one can make use of the upper limits on the distortions of the spectrum of the microwave background radiation to set limits on the heating of the intergalactic medium due to dissipation of turbulence for $z \gtrsim 10^{4}$. Sunyaev and Zeldovitch (1970) have thus found that for $z_{a} \sim 10^{4} \Omega^{-1 / 2}$ the energy input $\varepsilon \sim(\Delta u)^{3} / l \mathrm{erg} \mathrm{g}^{-1} \mathrm{~s}^{-1}$ is constrained by

$$
\Delta u / c<0.15 \Omega^{7 / 12}\left(l_{a} / c t_{a}\right)^{1 / 3}
$$

(where $l$ and $\Delta u$ are characteristic size and velocity changes for the largest eddies, $t_{a}$ corresponds to the epoch $z_{a}$, and $\left.\Omega=\varrho / \varrho_{\text {critical }}\right)$. But in Batchelor's theory

$$
B^{2} / 8 \pi \lesssim \frac{1}{2} \varrho c^{2} \sqrt{\varepsilon v / c^{4}}
$$

Thus for a fully ionized hydrogen plasma with $v \sim 10^{7} T^{5 / 2} / n$ one finds $\sqrt{\varepsilon v / c^{4}} \approx 10^{-12}$. But after $z \sim 10^{3}$, the turbulent velocity spins down as $z^{-2}$. Thus for a closed universe, 
$B_{0} \lesssim 10^{-14} \mathrm{G}$. A simple energetic upper limit, based on the maximum allowable energy release at $z \sim 10^{4}$ of $4 \times 10^{-3}$ of the radiation energy density then, sets an upper limit of $B_{0} \sim 10^{-9} \mathrm{G}$.

As has been pointed out by Kraichnan and Nagarjan (1967), it is at present unclear which limit (if in fact either) is applicable to a turbulently generated magnetic field in an incompressible fluid. Biermann and Schluter (1952) have argued that the fluid is amplified until its energy density is comparable to that of the turbulent medium. Batchelor (1950), making an analogy between $B$-fields and vorticity, has argued that only the smallest turbulent eddies support the field. In fact, the very existence of turbulent dynamos under these conditions is in doubt. Thus the order of magnitude of the asymptotic growth or decay rate can be roughly determined; but not which of the two will occur. Though a discussion of these questions is not appropriate here, they should be kept in mind in considering positive estimates of primordial magnetic fields.

In passing we remark that the limit on $B$-fields associated with the alignment of baryon or lepton dipole moments in the early universe which are subsequently supported by currents is of order $10^{-14} \mathrm{G}$. Magnetic monopoles, which cannot have a net non-zero value in a closed universe (noting that $\nabla \cdot \mathbf{B}=4 \pi \varrho_{M}$, using Gauss's theorem, and integrating over a closed surface), are not obviously eliminated in other models. Further examination of a monopole origin for cosmic $B$-fields does not at present seem fruitful.

\section{Intergalactic $B$-Fields}

The final possible source of primordial magnetic fields which has received attention of late is the source free spatially homogeneous $B$-field. Its existence in the past cannot be theoretically argued away. It has been speculated that this field may be associated with an anisotropic universe. The present upper limits on the $12 \mathrm{hr}$ anisotropy of the universe from measurements of fluctuations in the temperature of the microwave background radiation over the sky do not suffice to rule out even a relatively large intergalactic field of order $10^{-9} \mathrm{G}$. Its presence as a component of the intergalactic medium can be severely constrained, however, by examining the rotation measures of distant radio sources. If quasars are at cosmological distances, then from their observed rotation measures an upper limit on a magnetic field homogeneous out to $z \sim 2$ can be set. Even if they are not, strong radio galaxies can be used to set a limit which is only slightly weaker out to $z \sim 0.1$ or 0.2 . In a universe with deceleration parameter $q_{0}$, cosmological constant $\Lambda=0$, Hubble constant $H_{0}$, ionized intergalactic hydrogen density $n_{0}$, and local magnetic field $B_{\|, 0}$ we find that

$$
R M=\frac{e^{3} H_{0}^{-1} B_{\|, 0} n_{0}}{12 \pi m_{e}^{2} c^{3} q_{0}^{2}}\left\{\left(1+2 q_{0} z\right)^{3 / 2}+\left(1+2 q_{0} z\right)^{1 / 2}\left(6 q_{0}-3\right)+2-6 q_{0}\right\} .
$$

To determine the intergalactic contribution to the rotation measures of sources observed, one must subtract out both the intrinsic and our galactic contributions. 
Considering only high galactic latitude $\left(b^{\mathrm{II}} \geq 35^{\circ}\right)$, we estimate that the contribution to the rotation measure is less than $30 \mathrm{rad} / \mathrm{m}^{-2}$ for $z \approx 2$ (and $\lesssim 10 \mathrm{rad} / \mathrm{m}^{-2}$ for $z \sim 0.2$ ). For $q_{0}=\frac{1}{2}$ and $n_{0}=10^{-5}$, the magnetic field $B_{0} \lesssim 3 \times 10^{-10} \mathrm{G}\left(B_{0} \lesssim 1 \times 10^{-9} \mathrm{G}\right.$ for $z=0.2$ ), too small to account for the galactic fields. For an open universe with the observed parameters, the result is not incompatible with the observed galactic fields. However, if there were a large scale homogeneous $B$-field, one would expect to find a correlation between $z \cos \theta$ and $R M$ for distant sources where $\theta$ is the angle between $B$ and the direction of the source. Using the most complete list of rotation measures of extragalactic radio sources (Gardner et al., 1969), no statistically significant correlation is found between $R M$ and either $z$ or $z \cos \theta$; thus it is doubtful that any such correlations (and homogeneous $B$-fields) exist.

The presence of an inhomogeneous intergalactic magnetic field at present is more difficult to determine. However, if, for example the X-ray background radiation is due to Compton scattering of electrons on the microwave background radiation in intergalactic space, a lower limit on such a field necessary to isotropize the electrons can be set (depending on its topology). An upper limit of $B_{0} \lesssim 10^{-8} \mathrm{G}$ can be set from the maximum allowable non-thermal radio background these electrons would produce in the intergalactic medium.

\section{Dynamics of the $B$-Fields in Galaxies}

If there were a small, intergalactic $B$-field (either inhomogeneous on a galactic scale, or one more homogeneous with $R_{\text {protogalaxy }} \times|\nabla B| B \mid \ll 1$ ), it has been often stated that differential rotation should wind up, and thus amplify, the field after galaxy formation. Since $\tau \sim 2 \times 10^{8} \mathrm{yr}$, one might think that magnetic fields must then be increased by a factor of order 50 in a Hubble time, and thus that the above limits would not rule out a primordial origin to the fields. However, the resulting tightly wound field seems to be in conflict with observations of galactic field structure derived from Faraday rotation and interstellar polarization observations of our Galaxy, and from nonthermal radio observations of $\mathrm{M} 31$.

In addition it is not clear that magnetic fields are simply 'frozen' into the collapsing protogalaxy. For example, if the pre-galactic matter is in turbulent motion, magnetic fields may be able to diffuse out because of enhanced ambipolar diffusion. If as Parker has suggested (1970) the diffusivity is as high as $\eta \approx 10^{26} \mathrm{~cm}^{2} \mathrm{~s}^{-1}$, then when the protogalaxy is oblate and has reached a half thickness $L \approx 1 \mathrm{kpc}$, the leakage lifetime will be $\tau_{L} \sim L^{2} / \eta \lesssim H_{0}^{-1}$. On the other hand, such turbulence might tend to amplify the primordial seed field via the turbulent dynamo mechanism, or reduce it even further by enhancing the dissipative processes.

To complicate matters still further, one must consider the dynamical effects of the cosmic ray-magnetic field interaction since the galaxy has formed. The magnetic fields lying parallel to the galactic disc will be subject to Rayleigh-Taylor instability. Thus the pressure of the cosmic rays can cause the field at a weaker than average point to expand out of the disc. If magnetic 'bubbles' form, they may either contribute to a 
galactic 'halo' (the existence of which is in great doubt), or may pinch off from the general galactic field and be lost to intergalactic space. In this way, intergalactic fields arise, both out of normal galaxies and out of strong radio sources and quasars. However, it is not clear that this should give rise to a net loss of magnetic flux in the galaxy (the number of lines remaining constant in the disk), the ejected $B$-field energy essentially being supplied by the work done by cosmic rays in leaving the galaxy. Other processes also play a role in amplifying and destroying the galactic fields once they are present which will not be considered here.

\section{Origin of Galactic $B$-Fields and Conclusions}

Despite the complications galaxies present as magnetic fields are condensed into them, we are led to conclude that there are good reasons to believe that galactic magnetic fields are not primordial (and no good reasons to suppose that they are) and, therefore, that they are produced in the galaxies themselves. Aside from Harrison's picture, there seem to be only two current offerings for a galactic origin of these fields. Parker (1971) has suggested that magnetic fields are due to cyclonic turbulence in interstellar space, driven by the rotation of the galaxy (which is the ultimate source of the magnetic field energy). However, the mechanism depends for its success on the enhanced diffusion of the medium because of turbulence, which is as yet an unsettled matter. In addition, it does not direct itself specifically to the topology of the fields, but only to their magnitude.

A more intriguing possibility, and one which may have some observational support, is that the galactic magnetic fields, like the cosmic rays, are produced in energetically active regions such as pulsars or galactic nuclei. Current speculation considers both of these as some sort of dense, spinning object. In the case of the Crab, it is surely not a coincidence that the otherwise unexplainable flux of the entire nebula $\left(\sim 10^{34} \mathrm{G} \mathrm{cm}^{-2}\right)$ is about $10^{12}$ times larger than that in a typical star, just the field amplification that would be produced in the lifetime of the Crab pulsar NP $0532(n \sim \omega t \sim 30 \times 3 \times$ $\times 10^{10} \sim 10^{12}$ ) if the magnetic field of the spinning neutron star were rotating against some non-corotating plasma. Thus the general galactic fields might simply be the superposition of old supernova remnants. So, too, the excessively large magnetic field (and cosmic ray flux and rapid expansion) of the galactic center region might owe its origin to one or more dense, rotating objects there. Similarly, the $B$-fields in strong radio sources and quasars which are certainly not primordial, might be associated with 'spinar' like objects and rotational magnetic field amplification. Instabilities in the magnetic fields of such objects could give rise to jets (M87) or to the double bubbles characteristic of strong radio sources. The dynamics of such field amplification, expansion, and instability are only now being investigated.

Altogether, these considerations reinforce the conclusions that it is unlikely that the origin of cosmic magnetic fields can be found in the early universe and that, conversely, the present intergalactic $B$-fields, as well as the galactic fields, owe their origin to galactic processes. 


\section{References}

Batchelor, G. K.: 1950, Proc. Roy. Soc. London, 201 A, 405.

Biermann, L. and Schluter, A.: 1951, Phys. Rev. 82, 863.

Brecher, K. and Blumenthal, G. R.: 1970, Astrophys. Letters 6, 169.

Gardner, F. F., Morris, D., and Whiteoak, J. B.: 1969, Australian J. Phys. 22, 79.

Harrison, E. R.: 1970, Monthly Notices Roy. Astron. Soc. 148, 119.

Kraichnan, R. H. and Nagarajan, S.: 1967, Phys. Fluids 10, 859.

Ozernoy, L. M. and Chernin, A. D.: 1968, Soviet Astron. 11, 1968.

Parker, E. N.: 1971, Astrophys. J. 163, 255.

Sunyaev, R. A. and Zeldovitch, Ya. B.: 1970, Astrophys. Space Sci. 7, 20.

Woltjer, L.: 1965, in The Structure and Evolution of Galaxies, Interscience, New York. 